\title{
Immunological imbalance between IFN- $\gamma$ and IL-10 levels in the sera of patients with the cardiac form of Chagas disease
}

\author{
Daniela A D'Ávila', Paulo MM Guedes²/+, Ana M Castro³, Eliane D Gontijo", Egler Chiari', \\ Lúcia MC Galvão ${ }^{1,5}$
}

\begin{abstract}
${ }^{1}$ Departamento de Parasitologia, Instituto de Ciências Biológicas ${ }^{4}$ Departamento de Medicina Preventiva e Social, Faculdade de Medicina, Universidade Federal de Minas Gerais, Belo Horizonte, Minas Gerais, Brasil ${ }^{2}$ Laboratório de Imunoparasitologia, Departamento de Bioquímica e Imunologia, Faculdade de Medicina de Ribeirão Preto, Universidade de São Paulo, Av. Bandeirantes 3900, 14049-900 Ribeirão Preto, SP, Brasil ' ${ }^{3}$ epartamento de Microbiologia, Imunologia, Parasitologia e Patologia, Instituto de Patologia Tropical e Saúde Pública, Universidade Federal de Goiás, Goiânia, Brasil ${ }^{5}$ Programas de Pós-Graduação de Ciências da Saúde e Ciências Farmacêuticas, Centro de Ciências da Saúde, Universidade Federal do Rio Grande do Norte, Natal, Rio Grande do Norte, Brasil
\end{abstract}

The immune response is crucial for protection against disease; however, immunological imbalances can lead to heart and digestive tract lesions in chagasic patients. Several studies have evaluated the cellular and humoral immune responses in chagasic patients in an attempt to correlate immunological findings with clinical forms of Chagas disease. Moreover, immunoglobulins and cytokines are important for parasitic control and are involved in lesion genesis. Here, cytokine and IgG isotype production were studied, using total epimastigote antigen on sera of chagasic patients with indeterminate (IND, $n=27)$ and cardiac (CARD, $n=16)$ forms of the disease. Samples from normal, uninfected individuals (NI, $n=30$ ) were use as controls. The results showed that sera from both IND and CARD patients contained higher levels of Trypanosoma cruzi-specific IgG1 (IgGI) antibodies than sera from NI. No difference in IgG2 production levels was observed between NI, IND and CARD patients, nor was a difference in IL-10 and IFN- $\gamma$ production detected in the sera of IND, CARD and NI patients. However, IND patients displayed a positive correlation between IL-10 and IFN- $\gamma$ levels in serum, while CARD patients showed no such correlation, indicating an uncontrolled inflammatory response in CARD patients. These findings support the hypothesis that a lack of efficient regulation between IFN- $\gamma$ and IL-10 productions in CARD patients may lead to cardiac immunopathology.

Key words: Trypanosoma cruzi - Chagas disease - immunopathogenesis - cytokines - IgG isotype

It is well accepted that the host's immune response plays a key role during Trypanosoma cruzi infection, leading to either parasite control during the acute phase or to participation in the pathology development during the chronic phase (Dutra \& Gollob 2008). Antibody production and T-cell responses have been studied in patients with Chagas disease (Morgan et al. 1996, Cordeiro et al. 2001) and experimental models (Giordanengo et al. 2000, Guedes et al. 2008). Mononuclear cells from the heart tissue or peripheral blood of patients with the cardiac form of the disease produce higher IFN- $\gamma$ and TNF- $\alpha$ and lower or absent production of IL-10 and IL-4 compared with asymptomatic individuals (Correa-Oliveira et al. 1999, Ribeirao et al. 2000, Gomes et al. 2003). However, other studies have shown that the mean levels of mRNA expression for IL-5, IL-10, IL-13 and IFN- $\gamma$ were dramatically increased in peripheral blood mononuclear cells (PBMCs) freshly isolated from chagasic patients, regardless of the clinical form, compared to uninfected individuals (NI) (Dutra et al. 1997). Furthermore, the authors observed no

Financial support: CNPq (MCT/CNPq/MS-SCTIE-DECIT 25/2006 Estudo de Doenças Negligenciadas)

+ Corresponding author: pauloguedes@usp.br

Received 25 September 2008

Accepted 16 January 2009 significant differences in cytokine levels between cells from cardiac (CARD) and indeterminate patients (IND) upon stimulation with parasite-derived antigens (epimastigote and trypomastigote) (Dutra et al. 1997).

Similarly, cytokine levels in the sera from CARD and IND patients were analyzed and contradictory results were obtained. Higher TNF- $\alpha$ (Ferreira et al. 2003, Talvani et al. 2004) and NO levels (Perez-Fuentes et al. 2007) were observed in the plasma of chagasic CARD patients with the severe form of the disease, compared to patients with mild cardiopathy and NI. These results suggest a role for systemic NO and TNF- $\alpha$ in dilated cardiomyopathy. In contrast, no difference was observed in cytokine levels in the sera of chagasic patients with different clinical outcomes. IL- 2 , IFN- $\gamma$, and TNF- $\alpha$ production were similar in all groups of chagasic patients with different forms of the disease (Ward et al. 1999). These results were confirmed by other studies, which also showed that, although the levels of TNF- $\alpha$ and NO were higher in sera from chagasic patients, compared to non-chagasic individuals, no difference was observed between asymptomatic and symptomatic chagasic patients (Perez-Fuentes et al. 2008). IFN- $\gamma$ levels were similar in the sera of asymptomatic individuals and CARD patients that presented the form of the disease (Ribeirao et al. 2000). Thus, the findings of several studies have failed to clearly establish a correlation between the concentration of circulating cytokines and the cardiac form of Chagas disease. 
Studies concerning antibody production during the chronic phase of Chagas disease have focused on the analysis of $\operatorname{IgG}$ isotypes. Differences in the biological properties of these antibodies may have an important effect on the natural history of Chagas disease. Moreover, the production of different antibody isotypes is controlled by distinct cytokines. In chronic chagasic patients, $\operatorname{IgG} 1$ and $\operatorname{IgG} 2$ form about $90 \%$ of the $\operatorname{IgG}$ produced (Watthanakulpanich et al. 2008). It has been demonstrated that Th1 cytokines (IL-12, IFN- $\gamma$, TNF- $\alpha$ ) are responsible for the production of IgG1 and IgG3 isotypes, while Th2 cytokines (IL-4 and IL-10) stimulate IgG2 production (Briere et al. 1994, Kawano et al. 1994). IgG1 mediates lysis binding to complement $\mathrm{Clq}$ protein and macrophage phagocytosis, while IgG2 mediates immunity by non-phagocytic effectors cells (Fanger et al. 1991). Correlation between the different clinical forms of Chagas disease and the levels of $\operatorname{IgG}$ isotypes has not been clearly defined. Several authors have tried to demonstrate the correlation between $\operatorname{IgG}$ isotypes and the severity of the different clinical forms of Chagas disease both in patient groups (Cerban et al. 1993, Morgan et al. 1996, Michailowsky et al. 2003) and in experimental models (Giordanengo et al. 2000, Guedes et al. 2008). While some studies detected no differences between the levels of these immunoglobulins (IgG) among individuals with different clinical manifestations (Cerban et al. 1993, Michailowsky et al. 2003), others demonstrated higher levels of IgG2 antibodies in the sera of patients with cardiac and digestive manifestations of the disease (Morgan et al. 1996, Cordeiro et al. 2001).

The aim of the present study was to analyze the levels of IL-10, IFN- $\gamma$ and anti-T. cruzi antibody (IgG1 and $\mathrm{IgG} 2$ isotypes) production and determine the possible usefulness of these cytokines and antibodies as immunological markers of clinical evolution in the sera of chagasic IND and CARD patients with clinical forms of the disease. Correlation analysis between regulatory and inflammatory cytokine production in CARD and IND patients was performed to improve the current understanding of the immunoregulation involved in pathological development in human Chagas disease. Analysis of the results showed an imbalanced immune response in CARD patients, suggesting that an uncontrolled immune response is associated with cardiac disease.

\section{PATIENTS AND METHODS}

Patients - The inclusion of all subjects in the present investigation was approved by two independent Ethical Committees [Universidade Federal de Minas Gerais (087/99), Belo Horizonte, Minas Gerais, and Hospital São Salvador, Goiânia, Goiás, Brazil]. Signed, informed consent forms were obtained from the participants. All patients $(n=43)$ showed positive serology for Chagas disease, as determined by ELISA and immunofluorescence tests (Camargo 1966). The patients were from endemic areas in Brazil. The data (number, sex, age and clinical form) are summarized in Table. Based on their clinical records, the chagasic patients were divided into two different groups, IND and CARD. Patients presenting asymptomatic T. cruzi infection, classified as IND
TABLE

Age, sex and clinical forms of chronic chagasic patients from states of Minas Gerais, Goiás and Mato Grosso, central region of Brazil

\begin{tabular}{|c|c|c|c|}
\hline $\begin{array}{l}\text { Patient } \\
\text { number }\end{array}$ & Sex & $\begin{array}{c}\text { Age } \\
\text { (years) }\end{array}$ & $\begin{array}{c}\text { Clinical } \\
\text { form }\end{array}$ \\
\hline 1 & $\mathrm{~F}$ & 27 & IND \\
\hline 2 & M & 51 & IND \\
\hline 3 & M & 45 & CARD \\
\hline 4 & $\mathrm{~F}$ & 33 & IND \\
\hline 5 & M & 33 & IND \\
\hline 6 & $\mathrm{~F}$ & 27 & IND \\
\hline 7 & $\mathrm{~F}$ & 39 & IND \\
\hline 8 & M & 25 & IND \\
\hline 9 & M & 44 & CARD \\
\hline 10 & $\mathrm{~F}$ & 46 & IND \\
\hline 11 & $\mathrm{~F}$ & 54 & CARD \\
\hline 12 & $\mathrm{~F}$ & 49 & CARD \\
\hline 13 & $\mathrm{~F}$ & 54 & IND \\
\hline 14 & $\mathrm{~F}$ & 35 & CARD \\
\hline 15 & $\mathrm{~F}$ & 31 & CARD \\
\hline 16 & $\mathrm{~F}$ & 37 & IND \\
\hline 17 & $\mathrm{~F}$ & 26 & IND \\
\hline 18 & $\mathrm{~F}$ & 44 & IND \\
\hline 19 & $\mathrm{~F}$ & 43 & IND \\
\hline 20 & $\mathrm{~F}$ & 33 & IND \\
\hline 21 & $\mathrm{~F}$ & 20 & IND \\
\hline 22 & M & 30 & IND \\
\hline 23 & M & 42 & IND \\
\hline 24 & $\mathrm{~F}$ & 22 & IND \\
\hline 25 & M & 30 & IND \\
\hline 26 & M & 38 & IND \\
\hline 27 & $\mathrm{~F}$ & 35 & IND \\
\hline 28 & $\mathrm{~F}$ & 53 & IND \\
\hline 29 & $\mathrm{~F}$ & 33 & CARD \\
\hline 30 & M & 42 & IND \\
\hline 31 & $\mathrm{~F}$ & 44 & IND \\
\hline 32 & $\mathrm{~F}$ & 40 & IND \\
\hline 33 & M & 50 & CARD \\
\hline 34 & M & 75 & IND \\
\hline 35 & M & 57 & CARD \\
\hline 36 & M & 45 & CARD \\
\hline 37 & $\mathrm{~F}$ & 39 & CARD \\
\hline 38 & $\mathrm{~F}$ & 50 & CARD \\
\hline 39 & $\mathrm{~F}$ & 59 & CARD \\
\hline 40 & M & 25 & CARD \\
\hline 41 & M & 57 & CARD \\
\hline 42 & M & 76 & IND \\
\hline 43 & $\mathrm{~F}$ & 47 & CARD \\
\hline
\end{tabular}

CARD: cardiac; F: female; IND: indeterminate; M: male.

$(n=27)$, presented no clinical manifestations of the disease other than their positive serology. Patients with cardiac dysfunction $(n=16)$ presented with dilated cardiomyopathy and were identified via a detailed clinical examination, including electrocardiogram, chest Xray, Holter and echodopplercardiography. Sera from NI $(\mathrm{n}=30)$ were obtained from seronegative healthy inhab- 
itants of the same endemic areas. Normal individuals did not differ from Chagas disease patients with regard to age or gender.

Conventional serology (ELISA) - Peripheral blood was collected and maintained at $4^{\circ} \mathrm{C}$ for $30 \mathrm{~min}$ to promote clot formation. Serum samples were stored at $-80^{\circ} \mathrm{C}$ prior to cytokine quantification. ELISA tests were performed according to the methods of Voller et al. (1976). Briefly, ELISA plates were sensitized with $T$. cruzi antigen prepared by alkaline extraction of the $\mathrm{Y}$ strain, obtained by exponential growth in LIT medium. The sera were added to the plates and antibody binding was detected using peroxidase-labeled anti-human IgG. Total IgG or IgG1 and IgG2 isotypes conjugated to horseradish peroxidase (Bethyl Laboratories, Montgomery, USA) were used to determine antibody levels. The plates were read in a spectrophotometer using a $490 \mathrm{~nm}$ filter (BIO-RAD, 3550). The cut-off was determined using the mean absorbance of $10 \mathrm{NI}$ individuals plus two standard deviations.

Cytokine quantification (ELISA) - Cytokine levels in the sera were determined by ELISA. The ELISA sets were IL-10 and IFN- $\gamma$ (R \& D Duoset, R \& D, Minneapolis, $\mathrm{MN}$ ). Procedures were performed according to the manufacturer's instructions. The reaction was detected by peroxidase-conjugated streptavidin followed by a substrate mixture containing hydrogen peroxide and ABTS (Sigma Aldrich, St. Louis, MO) as a chromogen.

Statistical analyses - Regression analysis was used to compare antibody and cytokine levels; regression lines were compared by analysis of covariance. In all cases, differences were considered statistically significant when $\mathrm{p}<0.05$. All analyses were performed using the PRISM 3.0 software (GraphPad, San Diego, CA).

\section{RESULTS}

In order to correlate clinical findings with the immunological response observed during chronic Chagas disease, and to identify potential immunological pathogenesis markers, circulating cytokine (IL-10 and IFN- $\gamma$ ) and $\mathrm{IgG}$ isotype (total $\mathrm{IgG}$, IgG1 and IgG2) levels were determined in IND and CARD chagasic patients.
Higher production of total IgG was observed in IND $(1.26 \pm 0.17)$ and CARD $(1.23 \pm 0.13)$ patients compared to NI $(0.18 \pm 0.04, p<0.05)$ patients (Fig. 1A). An analysis of the results showed that sera from both IND $(0.86$ $\pm 0.23)$ and CARD $(0.88 \pm 0.22)$ patients presented with higher levels of $T$. cruzi-specific IgG1 antibodies than NI $(0.14 \pm 0.03, \mathrm{p}<0.05)$ patients. However, no difference was observed between IND and CARD patients in terms of IgG1 production (Fig. 1B). No difference was observed in IgG2 levels between IND $(0.34 \pm 0.14)$, CARD $(0.32 \pm$ $0.16)$ and NI $(0.23 \pm 0.09)$ individuals (Fig. 1C).

Statistically similar levels of IL-10 were detected in the sera of IND patients $(9.48 \pm 3.09)$, CARD chagasic patients $(13.68 \pm 2.35)$ and NI individuals $(8.13 \pm 3.82)$ (Fig. 2). Patients with IND $(59.44 \pm 18.63)$ and CARD $(80.12 \pm$ 22.32) forms of Chagas disease, as well as NI individuals (53.83 \pm 17.41$)$ presented similar IFN- $\gamma$ levels (Fig. 2).

In an attempt to clarify the immunoregulatory mechanisms involved in the chronic cardiac pathology of Chagas disease, our group investigated whether a correlation exists between cytokine and $\mathrm{IgG}$ isotype production, and between regulatory (IL-10) and inflammatory (IFN- $\gamma$ ) cytokine levels in CARD and IND patients. No correlation was observed between cytokine (IL-10 and IFN- $\gamma$ ) and $\mathrm{IgG}$ isotype (IgG1 and IgG2) production (data not shown); however, a positive correlation $(\mathrm{R}=0.82$, $\mathrm{p}<0.0001$ ) (Fig. 3A) was observed between IFN- $\gamma$ and IL-10 levels in the sera of IND patients. Interestingly, an analysis of CARD patients showed no correlation $(\mathrm{R}=0.11, \mathrm{p}=0.69)$ between regulatory and inflammatory cytokine production (Fig. 3B).

\section{DISCUSSION}

Distinct cytokine profiles exert important influences in IgG production, and antibodies are important to control parasite infection and immunopathological processes (Tibbetts et al. 1994). The current results, using total epimastigote antigen, showed high levels of IgG1 in chagasic patients compared to non-chagasic individuals and similar IgG1 levels in with the IND versus the CARD clinical forms. These results are in agreement with data that demonstrated high IgG1 concentrations against epimastigote antigen in sera from all groups of chagasic pa-
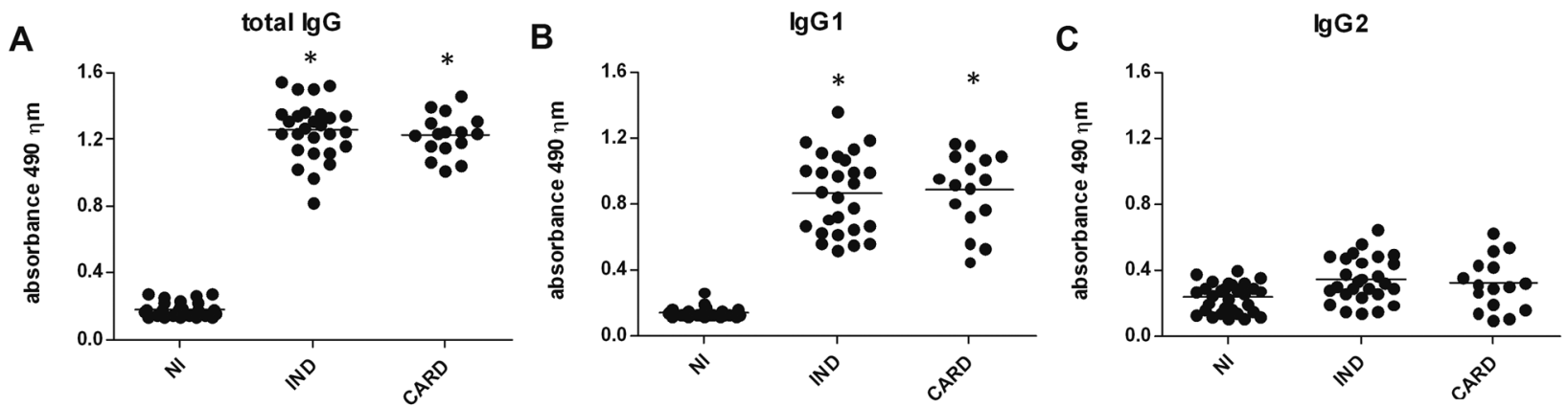

Fig. 1: Trypanosoma cruzi specific total-IgG (A), IgG1 (B) and IgG2 (C) antibodies in the sera of indeterminate (IND) and cardiac (CARD) patients with chronic Chagas disease and noninfected individuals (NI). Asterisks indicate statistic significance for comparisons between NI and chagasic patients (IND and CARD) $(\mathrm{p}<0.05)$. Each data point represents the mean absorbance of duplicate wells. 


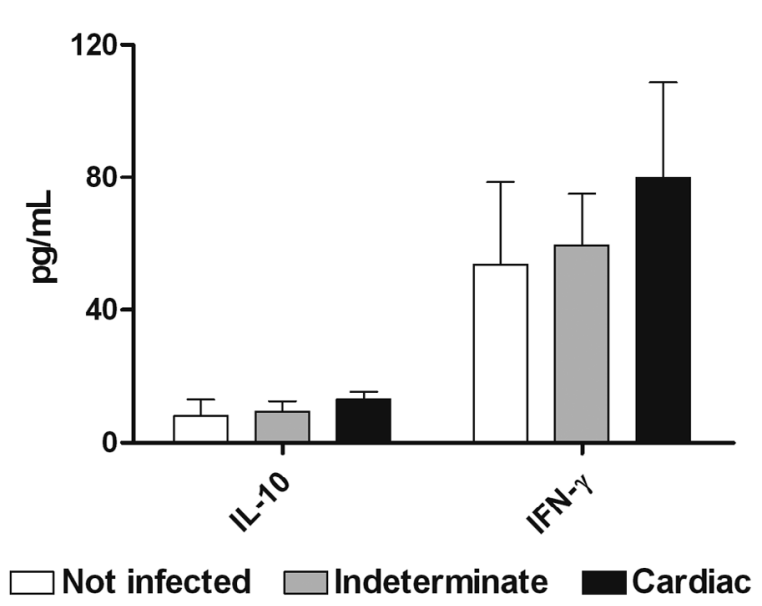

Fig. 2: IL-10 and IFN- $\gamma$ quantification by ELISA in the sera of indeterminate and cardiac patients with chronic Chagas disease and non infected individuals. Results are mean \pm standard error medium.

A



B

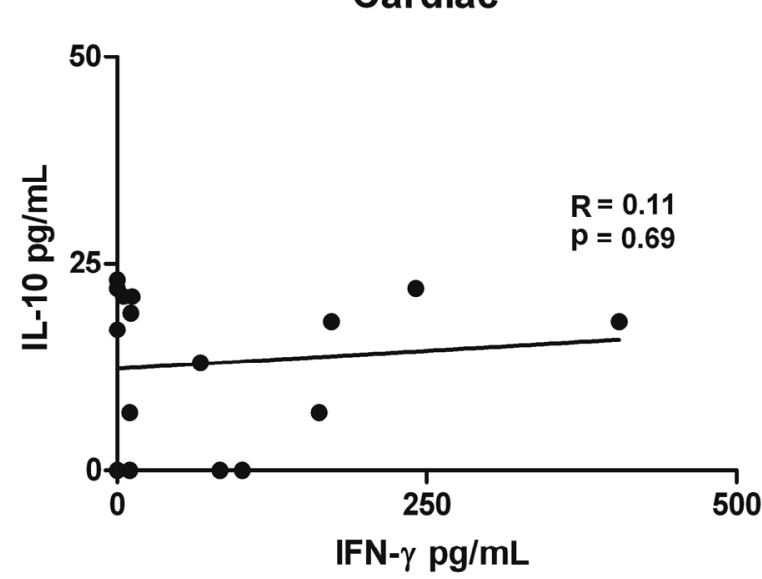

Fig. 3: correlation between IFN- $\gamma$ and IL-10 levels in the sera of chagasic patients that presented the indeterminate (A) and cardiac (B) forms of the disease. Positive correlation was considered significant when the $\mathrm{p}<0.05$. tients by ELISA, regardless of the clinical form (Cerban et al. 1993). High IgG1 and similar IgG3 production in patients presenting different clinical forms of the disease were described using different antigenic preparations, such as paraflagellar rod protein (Michailowsky et al. 2003). Cerban et al. (1993) observed no significant differences regarding IgG isotype production in chronic chagasic patients with different clinical forms. However, a different antigenic recognition pattern by IgG1 among different clinical groups by immunoblotting using acidic antigenic fraction (AAF) separated from T. cruzi cytosol was observed. It seems that some relationship exists between the pattern of IgG1 reactivity with AAF and the degree of heart damage. The distinct production of $\mathrm{IgG}$ isotypes identified via ELISA was described for chagasic patients with different clinical forms of the disease. Analysis of literature data shows that when using epimastigote antigenic preparation, CARD patients produced elevated levels of IgG2 compared with the IND and NI groups (Morgan et al. 1996). It is possible that the preferential induction of different subsets of $\mathrm{T}$ helper cells by specific antigen or host genetic background may be crucial to the pattern of antibody response and, subsequently, in the immunopathology of Chagas disease. Patients with different clinical forms of Chagas disease have the ability to produce similar antibody levels; therefore, given the clinical and immunological differences among the groups, it is reasonable to hypothesize that these antibodies may recognize different antigenic targets.

Cytokine profiling has been demonstrated as fundamental to defining the immunopathological mechanisms involved in chronic chagasic cardiomyopathy and controlling the immune response during $T$. cruzi infection (Zhang \& Tarleton 1996). The proinflammatory cytokines IL-12, IFN- $\gamma$ and TNF- $\alpha$ (Th1 response) act in concert to activate macrophages to kill the parasites through the production of nitric oxide and NO-derived nitrogen free radicals. In addition, they also stimulate the differentiation and proliferation of Th1-biased CD4 $\mathrm{T}$ cells, which may orchestrate a CD8 $\mathrm{T}$ cell response that causes tissue destruction and fibrosis (Higuchi et al. 2003). As expected, this inflammatory response needs to be regulated, and this occurs mainly via the action of the anti-inflammatory cytokines IL-4, IL-10 and TGF- $\beta$ (Silva et al. 1992, Holscher et al. 1998). Thus, cytokine expression may be correlated with clinical manifestations of disease.

A few studies concerning cytokine sera levels have been performed in individuals with different clinical forms of Chagas disease and contradictory results were obtained. It is possible that the contradictions are due to the procedures used for sample collection, which can influence the outcome of laboratory measurements. This study showed that serum cytokine levels in patients with the CARD chronic form of the disease did not differ from those of IND patients. Thus, the present results are in agreement with the finding of Ward et al. (1999) that similar levels of IFN- $\gamma$ and TNF- $\alpha$ can be found in the sera of chronic chagasic patients (both IND and CARD form) and NI patients. Interestingly, although these authors used different collection procedures than 
those used by our group, similar results were obtained. This implies that other factors, possibly related to the patient classification criteria, could also be involved, as suggested by Dutra et al. (2005). Similar high levels of TNF- $\alpha$ were detected in asymptomatic individuals and patients with digestive and CARD clinical forms, respectively (Perez-Fuentes et al. 2007). However, Ferreira et al. (2003) showed that patients with significant left ventricular (LV) dysfunction (LV ejection fraction $\leq 50 \%$ ) showed higher levels of TNF- $\alpha$, compared to chagasic patients without LV dysfunction. It has been suggested that chronic TNF- $\alpha$ production prior to heart failure may play a role in chronic Chagas disease cardiomyopathy progression (Ferreira et al. 2003). A similar level of cytokine production in sera of acute chagasic children, compared to NI, has been described previously (Moretti et al. 2002). Although the present results showed no differences between IL-10 and IFN- $\gamma$ levels in CARD and IND patients, the IND patients displayed a positive correlation between inflammatory and regulatory cytokine production, indicating a controlled immune response. These findings are supported by the higher production of IFN- $\gamma$ and TNF- $\alpha$ associated with high IL-10 levels described in IND patients, while CARD patients showed an unregulated Th1 response (Bahia-Oliveira et al. 1998, Correa-Oliveira et al. 1999, Ribeirao et al. 2000, Abel et al. 2001, Gomes et al. 2005). A lack of co-regulation between inflammatory and anti-inflammatory cytokines associated with severe disease was also demonstrated in human patients with leishmaniasis (Gaze et al. 2006), strengthening this hypothesis.

In conclusion, these findings suggest that no correlation exists between cytokine (IL-10 and IFN- $\gamma$ ) and IgG isotype (IgG1 and IgG2) levels in the sera of chagasic patients with IND and CARD clinical forms of Chagas disease. However, analysis of the results reinforces the fact that more efficient regulation of IFN- $\gamma$ and IL-10 production occurs in IND patients, compared to CARD patients, suggesting that the maintenance of a balanced immune response is critical for arresting pathology establishment.

\section{ACKNOWLEDGMENTS}

To Dr Walderez Ornelas Dutra, for her critical reading and revision of the manuscript, and to Afonso da Costa Viana and Orlando Carlos Magno, from the Departamento de Parasitologia, Instituto de Ciências Biológicas, UFMG, for their technical assistance. PMMG and LMCG received postdoctoral and visitor researcher fellowships from $\mathrm{CNPq}$.

\section{REFERENCES}

Abel LC, Rizzo LV, Ianni B, Albuquerque F, Bacal F, Carrara D, Bocchi EA, Teixeira HC, Mady C, Kalil J, Cunha-Neto E 2001. Chronic Chagas' disease cardiomyopathy patients display an increased IFN-gamma response to Trypanosoma cruzi infection. J Autoimmun 17: 99-107.

Bahia-Oliveira LM, Gomes JA, Rocha MO, Moreira MC, Lemos EM, Luz ZM, Pereira ME, Coffman RL, Dias JC, Cancado JR, Gazzinelli G, Correa-Oliveira R 1998. IFN-gamma in human Chagas' disease: protection or pathology? Braz J Med Biol Res 31: 127-131.
Briere F, Servet-Delprat C, Bridon JM, Saint-Remy JM, Banchereau J 1994. Human interleukin 10 induces naive surface immunoglobulin $\mathrm{D}+(\mathrm{sIgD}+) \mathrm{B}$ cells to secrete $\operatorname{IgG1}$ and IgG3. J Exp Med 179: 757-762.

Camargo ME 1966. Fluorescent antibody test for the serodiagnosis of American trypanosomiasis. Technical modification employing preserved culture forms of Trypanosoma cruzi in a slide test. Rev Inst Med Trop Sao Paulo 8: 227-235.

Cerban FM, Gea S, Menso E, Vottero-Cima E 1993. Chagas' disease: IgG isotypes against Trypanosoma cruzi cytosol acidic antigens in patients with different degrees of heart damage. Clin Immunol Immunopathol 67: 25-30.

Cordeiro FD, Martins-Filho OA, Da Costa Rocha MO, Adad SJ, Correa-Oliveira R, Romanha AJ 2001. Anti-Trypanosoma cruzi immunoglobulin $\mathrm{G} 1$ can be a useful tool for diagnosis and prognosis of human Chagas' disease. Clin Diagn Lab Immunol 8: 112-118.

Correa-Oliveira R, Gomes J, Lemos EM, Cardoso GM, Reis DD, Adad S, Crema E, Martins-Filho OA, Costa MO, Gazzinelli G, Bahia-Oliveira LM 1999. The role of the immune response on the development of severe clinical forms of human Chagas disease. Mem Inst Oswaldo Cruz 94: 253-255.

Dutra WO, Gollob KJ 2008. Current concepts in immunoregulation and pathology of human Chagas disease. Curr Opin Infect Dis 21: $287-292$.

Dutra WO, Gollob KJ, Pinto-Dias JC, Gazzinelli G, Correa-Oliveira R, Coffman RL, Carvalho-Parra JF 1997. Cytokine mRNA profile of peripheral blood mononuclear cells isolated from individuals with Trypanosoma cruzi chronic infection. Scand J Immunol 45: 74-80.

Dutra WO, Rocha MO, Teixeira MM 2005. The clinical immunology of human Chagas disease. Trends Parasitol 21: 581-587.

Fanger MW, Segal DM, Romet-Lemonne JL 1991. Bispecific antibodies and targeted cellular cytotoxicity. Immunol Today 12: 51-54.

Ferreira RC, Ianni BM, Abel LC, Buck P, Mady C, Kalil J, CunhaNeto E 2003. Increased plasma levels of tumor necrosis factoralpha in asymptomatic/"indeterminate" and Chagas disease cardiomyopathy patients. Mem Inst Oswaldo Cruz 98: 407-411.

Gaze ST, Dutra WO, Lessa M, Lessa H, Guimaraes LH, Jesus AR, Carvalho LP, Machado P, Carvalho EM, Gollob KJ 2006. Mucosal leishmaniasis patients display an activated inflammatory T-cell phenotype associated with a nonbalanced monocyte population. Scand J Immunol 63: 70-78.

Giordanengo L, Maldonado C, Rivarola HW, Iosa D, Girones N, Fresno $\mathrm{M}$, Gea S 2000. Induction of antibodies reactive to cardiac myosin and development of heart alterations in cruzipain-immunized mice and their offspring. Eur J Immunol 30: 3181-3189.

Gomes JA, Bahia-Oliveira LM, Rocha MO, Busek SC, Teixeira MM, Silva JS, Correa-Oliveira R 2005. Type 1 chemokine receptor expression in Chagas' disease correlates with morbidity in cardiac patients. Infect Immun 73: 7960-7966.

Gomes JA, Bahia-Oliveira LM, Rocha MO, Martins-Filho OA, Gazzinelli G, Correa-Oliveira R 2003. Evidence that development of severe cardiomyopathy in human Chagas' disease is due to a Th1-specific immune response. Infect Immun 71: 1185-1193.

Guedes PM, Veloso VM, Gollob KJ, Afonso LC, Caldas IS, Vianna P, Lana M, Chiari E, Bahia MT, Galvao LM 2008. IgG isotype profile is correlated with cardiomegaly in Beagle dogs infected with distinct Trypanosoma cruzi strains. Vet Immunol Immunopathol 124: 163-168.

Higuchi ML, Benvenuti LA, Martins Reis M, Metzger M 2003. Pathophysiology of the heart in Chagas' disease: current status and new developments. Cardiovasc Res 60: 96-107. 
Holscher C, Kohler G, Muller U, Mossmann H, Schaub GA, Brombacher F 1998. Defective nitric oxide effector functions lead to extreme susceptibility of Trypanosoma cruzi-infected mice deficient in gamma interferon receptor or inducible nitric oxide synthase. Infect Immun 66: 1208-1215.

Kawano Y, Noma T, Yata J 1994. Regulation of human IgG subclass production by cytokines. IFN-gamma and IL-6 act antagonistically in the induction of human IgG1 but additively in the induction of IgG2. J Immunol 153: 4948-4958.

Michailowsky V, Luhrs K, Rocha MO, Fouts D, Gazzinelli RT, Manning JE 2003. Humoral and cellular immune responses to Trypanosoma cruzi-derived paraflagellar rod proteins in patients with Chagas' disease. Infect Immun 71: 3165-3171.

Moretti E, Basso B, Cervetta L, Brigada A, Barbieri G 2002. Patterns of cytokines and soluble cellular receptors in the sera of children with acute Chagas' disease. Clin Diagn Lab Immunol 9: 1324-1327.

Morgan J, Dias JC, Gontijo ED, Bahia-Oliveira L, Correa-Oliveira R, Colley DG, Powell MR 1996. Anti-Trypanosoma cruzi antibody isotype profiles in patients with different clinical manifestations of Chagas' disease. Am J Trop Med Hyg 55: 355-359.

Perez-Fuentes R, Lopez-Colombo A, Ordonez-Toquero G, GomezAlbino I, Ramos J, Torres-Rasgado E, Salgado-Rosas H, RomeroDiaz M, Pulido-Perez P, Sanchez-Guillen MC 2007. Correlation of the serum concentrations of tumour necrosis factor and nitric oxide with disease severity in chronic Chagas disease (American trypanosomiasis). Ann Trop Med Parasitol 101: 123-132.

Perez-Fuentes R, Torres-Rasgado E, Salgado-Rosas H, ZamoraGinez I, Sanchez-Guillen MC 2008. The anti-oxidant defence response in individuals with the indeterminate form of Chagas disease (American trypanosomiasis). Ann Trop Med Parasitol 102: $189-197$.
Ribeirao M, Pereira-Chioccola VL, Renia L, Augusto Fragata Filho A, Schenkman S, Rodrigues MM 2000. Chagasic patients develop a type 1 immune response to Trypanosoma cruzi transsialidase. Parasite Immunol 22: 49-53.

Silva JS, Morrissey PJ, Grabstein KH, Mohler KM, Anderson D, Reed SG 1992. Interleukin 10 and interferon gamma regulation of experimental Trypanosoma cruzi infection. J Exp Med 175: 169-174.

Talvani A, Rocha MO, Barcelos LS, Gomes YM, Ribeiro AL, Teixeira MM 2004. Elevated concentrations of CCL2 and tumor necrosis factor-alpha in chagasic cardiomyopathy. Clin Infect Dis 38: 943-950.

Tibbetts RS, McCormick TS, Rowland EC, Miller SD, Engman DM 1994. Cardiac antigen-specific autoantibody production is associated with cardiomyopathy in Trypanosoma cruzi-infected mice. J Immunol 152: 1493-1499.

Voller A, Bidwell DE, Bartlett A 1976. Enzyme immunoassays in diagnostic medicine. Theory and practice. Bull World Health Organ 53: 55-65.

Ward LS, Guariento ME, Fernandes GA, Maciel RM 1999. Serum cytokines in chronic Chagas' disease. Rev Soc Bras Med Trop 32: $285-289$.

Watthanakulpanich D, Smith HV, Hobbs G, Whalley AJ, Billington D 2008. Application of Toxocara canis excretory-secretory antigens and IgG subclass antibodies (IgG1-4) in serodiagnostic assays of human toxocariasis. Acta Trop 106: 90-95.

Zhang L, Tarleton RL 1996. Characterization of cytokine production in murine Trypanosoma cruzi infection by in situ immunocytochemistry: lack of association between susceptibility and type 2 cytokine production. Eur J Immunol 26: 102-109. 